\title{
PROLONGED VISUAL FAILURE FOLLOWING CARDIAC ARREST DUE TO ACCIDENTAL HYPOTHERMIA*
}

\author{
BY
F. D. MCAULEY
Dublin

\section{Case Report}

A boy aged 2 years and 7 months fell through the ice into a flooded quarry on February 27, 1963. He was not breathing when taken from the water at 3.45 p.m., and mouth-to-mouth breathing was performed on the way to Our Lady's Hospital for Sick Children, Dublin, where he was admitted at 3.50 p.m.

Examination.-He was not breathing and the heart sounds could not be heard; the pupils were constricted and the body temperature (taken 5 minutes later) was only $88^{\circ} \mathrm{F}$.

Treatment.-Attempts at resuscitation by external cardiac massage were commenced by the anaesthetist (Dr. W. S. Wren) almost immediately, and heart beats became audible 10 minutes later at 4 p.m.

Progress.-Within 2 hours the patient was in a state of cerebral irritation, and the next day he showed response to painful stimuli.

February 28.-E.E.G. (Dr. N. V. O'Donohoe) Unconscious; satisfactory record. At normal gain, very little electrical activity could be detected in any area. With increased gain, slow irregular waves were seen bilaterally.

February 29.-On the third day he was opening his eyes and attempting to talk (in Irish-a language with which he was more familiar than English-his mother being a native speaker).

March 2.-E.E.G. Satisfactory. The record shows a remarkable improvement with the presence of regular medium voltage 5-6 c/s activity in all areas. No focal or lateralizing signs.

March 4.-7 days after the accident, when he was fully conscious, it was obvious that he could not see. His pupils were small and apparently reacting to light but his attention was not attracted by any object or by a light source. At this stage, however, he was quite cheerful and co-operative.

March 7.-E.E.G. Satisfactory record. The tracing does not show any further improvement and may show rather more slow activity than the previous record. In the central and anterior areas 4-6 c/s activity is well established. In the posterior areas, particularly on the right, there is still flattening, and irregular delta waves are present. This change was also present in the previous tracing.

CONCLUSION.-There is persistent flattening in the occipital areas, more marked on the right.

March 27.-On 24th day after the accident, ophthalmic examination revealed no apparent visual improvement; although quite oblivious of visual stimuli he was bright and cheerful.

April 14.-6 weeks after the accident his parents noticed that he was starting to find his way around and that he was attempting to pick up objects. The following week at an ophthalmic examination in the OutPatients' Department he was seen to get around very well, and could pick up objects easily and accurately.

April 26.-E.E.G. Fairly co-operative, satisfactory record. A further improvement was demonstrated in this record and regular 5-6 c/s activity was a feature in the occipital areas bilaterally, where voltage was also normal. The impression was that the improvement was somewhat better on the left than on the right.

CONCLUSION.-Further improvement. There is a residual doubtful abnormality in the right occipital area but the flattening, previously described, has disappeared.

Follow-up. -6 months later, in October, 34 weeks after the accident, ophthalmic examination showed that there was no apparent visual defect. He was now a very active and energetic child.

* Received for publication November 26, 1963. 
It was not possible-because of his playfulness-to measure his visual acuity with reasonable accuracy, but he did volunteer (when not asked to co-operate) that he could see a railway engine on a card, 6 metres away; this was equivalent to an acuity of 6/60. He could also see a $6 \mathrm{~d}$. piece on a varnished wooden floor from at least 6 feet. There was no visual field defect demonstrable by a light source. His optic discs were of good colour and his eyes appeared normal in every way.

\section{Discussion}

It is probable that the heart had been arrested for much longer than the accepted 4 minutes maximum, which is consistent with the expectation of full recovery of all functions. The hypothermia, therefore, must have been a factor facilitating recovery without obvious gross defect. (His body temperature was purposely kept at $96-97^{\circ} \mathrm{F}$. for the first few days after the accident.)

It will be noticed that his physical and mental improvement coincided with a marked change in his E.E.G. record on the third day, and that the E.E.G. record taken on the eighth day-when he was still apparently blind-showed no improvement on that taken 5 days earlier; it showed a persistent flattening in the occipital areas, more marked on the right side. An E.E.G. record taken 8 weeks after the accident, however, revealed an improvement on that taken on the eighth day, and it would appear that this concurred with the improvement in vision noted 2 weeks earlier. The cause of the child's temporary blindness would thus appear to have been localized in the occipital areas. In support of this conclusion is the fact that at no time- - either during the earlier stages of blindness or since-were there any abnormal ocular signs. The idea of occipital visual area disturbance is supported by a comparison of the E.E.G. tracings taken on the day of the accident, on the third day, on the eighth day, and after 8 weeks. The first, taken while the child was unconscious, stated that "very little electrical activity could be detected in any area". The second, taken on the third day when the child was conscious and attempting to speak, showed a "remarkable improvement with the presence of regular medium voltage 5-6 c/s activity in all areas". The third, taken on the eighth day, stated that "in the central and anterior areas 5-6 c/s activity was well established but in the posterior areasparticularly on the right side-there was still flattening and irregular delta waves were present". This change had also been present in the previous tracing and led to the conclusion of "persistent flattening in the occipital areas, more marked on the right". The record taken in the eighth week, however, when the child could see sufficiently well to get around and to pick up objects, showed regular 5-6 c/s activity in the occipital areas bilaterally where voltage was also normal, leading to the conclusion that the "flattening previously described had disappeared".

At no stage of his illness nor since have any other abnormal neurological signs been demonstrable apart from a doubtfully positive Babinski sign found on one occasion.

Dr. W. S. Wren applied the external cardiac massage and supervised subsequent resuscitative measures and Dr. N. V. O'Donohoe was responsible for the electro-encephalographic studies and for assessing the condition of the central nervous system. I am grateful for their co-operation and help in preparing this report. 